\title{
Statistical Methods for Clinical Trial Designs in the New Era of Cancer Treatment
}

\author{
Beibei Guo ${ }^{1 *}$ and Rui Zhang ${ }^{2}$ \\ ${ }^{1}$ Department of Experimental Statistics, Louisiana State University, USA \\ ${ }^{2}$ Department of Physics \& Astronomy, Louisiana State University, USA
}

Submission: January 30, 2018; Published: February 28, 2018

*Corresponding author: Beibei Guo, Department of Experimental Statistics, Louisiana State University, Baton Rouge, LA 70803, USA; Email: beibeiguo@lsu.edu

\begin{abstract}
Recent success of immunotherapy and other targeted therapies in cancer treatment has signaled the advent of precision medicine. Unlike conventional trial designs that aim to find an optimal treatment ignoring inter-patient heterogeneity, clinical trial designs for precision medicine must take into account patients' variability in genes, environments, and lifestyle. This article provides a review of recent research development of clinical trial designs toward this trend.
\end{abstract}

Keywords : Clinical trial designs; Precision medicine; Immunotherapy

\section{Introduction}

Traditional clinical trials aim to find an optimal treatment ignoring patient variability, which means the optimal treatment is the same for all patients of a specific disease. However, due to inter-patient heterogeneity, a treatment can be very successful for some patients but not for others. For example, some drugs may benefit only $2 \%$ of the patients and there are even drugs that are harmful to certain ethnic groups [1]. To overcome this "one-size-fits-all" approach and to acknowledge patient heterogeneity, a new 215-million national Precision Medicine Initiative was launched by former President Barack Obama in January 2015 [2]. Precision or personalized medicine provides emerging approaches for disease treatment and prevention that target to the needs of a patient based on his/her own individual characteristics such as biomarker, environmental exposures, lifestyle, and psychosocial characteristics. Therefore, precision medicine requires a departure from conventional clinical trial paradigms, in that the optimal treatment must be tailored to patient subgroups. This approach can yield treatment options for patients whose cancer is not responding favorably to the standard therapy.

Recently, immunotherapy is hailed as the biggest breakthrough for treating cancer since the first chemotherapies were developed [3-5]. It is an innovative treatment approach that harnesses and enhances the innate power of the immune system to fight cancer. The landmark success of immunotherapeutic agents such as ipilimumab, nivolumab and pembrolizumab has made immunotherapy a hot area in cancer research. Due to different mechanisms for treating cancer, immunotherapeutic agents behave differently than conventional chemotherapy and radiotherapy. For example, unlike traditional therapies, the efficacy of immunotherapeutic agents may not increase with the dose. In addition, besides toxicity and efficacy outcomes, immune response is an important outcome that measures the biological efficacy of the immunotherapeutic agent in activating the immune system. So it's critical in immunotherapy trials to incorporate the immune response into the trial design and leverage its close relationship with clinical endpoints. In addition, previous studies suggested that radiotherapy can generate significant antitumor immunity, and immunotherapeutic agents have been combined with radiotherapy in clinical trials to improve radiotherapy outcome [6-8]. For those trials, it is desirable to consider both radiation effect and potential overlapping toxicities of multiple modalities, and using biomarkers as surrogate endpoints of treatment efficacy and toxicity is highly encouraged $[9,10]$.

\section{Statistical methods for clinical trial designs in the new era}

A few clinical trial designs have been proposed in the literature to account for individual patient characteristics. For example, Piantadosi \& Liu [11] described a method that incorporates the patient's pharmacokinetic data into clinical trial designs. Babb \& Rogatko [12] proposed to utilize the patient's pretreatment concentration of an antibody in a clinical trial. 0 Quigley \& Paoletti [13], Yuan \& Chappell [14], and Ivanova \& Wang [15] presented designs for patient prognostic subgroups. 
Thall, et al. [16] proposed a trial design that accounts for patient characteristics and their interactions with the dose. These are very early attempts to select personalized treatments. However, due to the typical small sample sizes of early-phase clinical trials, these methods have a common limitation that they can accommodate very few patient characteristics in order to avoid the curse of dimensionality.

In practice, the number of patient characteristics that may affect the treatment effect can be large; and moreover, these characteristics may be highly correlated. To overcome these difficulties, Guo \& Yuan [17] developed a Bayesian dose-finding trial design that can accommodate a more realistic number of patient characteristics, which was motivated by a clinical trial of a targeted therapy in patients with locally advanced or metastatic solid tumors that may harbor molecular alterations in five genes. In their method, the canonical partial least squares (CPLS) [18] is employed to extract information from dose, patient characteristics, and dose-by-characteristics interactions. Based on the CPLS components, a latent-variable approach is used to model the ordinal toxicity and efficacy outcomes. This approach circumvents the curse of dimensionality and the potential high correlations among patient characteristics. The design has shown to have good operating characteristics through simulation studies.

The development of clinical trial designs for immunotherapy is still at its infancy. To the best of our knowledge, the only design published so far is the one by Liu, et al. [19] which is a Bayesian phase I/II dose-finding design that simultaneously considers three outcomes: immune response, toxicity, and efficacy. The trial objective is to identify the biologically optimal dose, defined as the dose with the highest desirability in the risk-benefit tradeoff. To reflect the fact that in immunotherapy, clinical responses rely on the activation of the immune system, the joint distribution of the three outcomes is factorized into the product of the marginal distribution of the immune response and the conditional distributions of efficacy and toxicity given the immune response. In an immunotherapy trial, the immune response is often scored as the change in a measure of immune activity from baseline to post-treatment, which is typically a continuous outcome. Efficacy and toxicity are often characterized as ordinal variables (complete response, partial response, stable disease, or progressive disease for efficacy; and grade 1, 2, 3, 4 adverse events for toxicity). Therefore, in the design of Liu, Guo, and Yuan, the Emax model is used to model the marginal distribution of the immune response; and conditional on the immune response, the joint distribution of toxicity and efficacy is modeled through a latent variable approach. Because this design is adaptive and explicitly accounts for patient heterogeneity in immune response, it can provide patients in the trial with better treatment regimes, thus greater safety and chance of therapeutic benefit.

\section{Conclusion}

The recent advances and rapid progress in precision medicine represent an unprecedented opportunity for the development of clinical trial designs that are customized for individual patients. In this article, we brie y review some new clinical trial designs that can accommodate the key features of precision medicine and immunotherapy. The development of novel trial designs for precision medicine continues to be an active and important area of statistical methodology research. For example, the designs we reviewed all assume that the patient outcomes are quickly ascertainable so that the decision rules can be applied when the new patient enters the trial. However, in a real trial, the outcomes may be evaluated after the first few treatment cycles. Incorporating late-onset outcomes is a future topic of interest. Recently, combining immunotherapy with chemotherapy or radiotherapy, combining two immunotherapeutic agents, and integration of molecularly targeted agents in radiotherapy have received tremendous attention in oncology; as a result, there is much ongoing research in these areas.

\section{References}

1. Schork NJ (2015) Personalized medicine: time for one-person trials. Nature 520(7549): 609-611.

2. Collins FS, Varmus H (2015) A new initiative on precision medicine. $\mathrm{N}$ Engl J Med 372(9): 793-795.

3. Topalian SL, Weiner GJ, Pardoll DM (2011) Cancer immunotherapy comes of age. J Clin Oncol 29(36): 4828-4836.

4. Couzin Frankel J (2013) Cancer immunotherapy. Science 324: 14321433.

5. Makkouk A, Weiner GJ (2015) Cancer immunotherapy and breaking immune tolerance: new approaches to an old challenge. Cancer Res 75(1): 5-10.

6. Crittenden M, Kohrt H, Levy R, Jones J, Camphausen K, et al. (2015) Current clinical trials testing combinations of immunotherapy and radiation. Semin Radiat Oncol 25(1): 54-64.

7. Daly ME, Monjazeb AM, Kelly K (2015) Clinical trials integrating immunotherapy and radiation for non-small-cell lung cancer. J Thorac Oncol 10(12): 1685-1693.

8. Kang J, Demaria S, Formenti S (2016) Current clinical trials testing the combination of immunotherapy with radiotherapy. J Immunother Cancer 4: 51 .

9. Freidlin B, McShane LM, Polley MY, Korn EL (2012) Randomized phase II trial designs with biomarkers. J Clin Oncol 30(26): 3304-3309.

10. Mc Shane LM, Hunsberger S, Adjei AA (2009) Effective incorporation of biomarkers into phase II trials. Clin Cancer Res 15(6): 1898-1095.

11. Piantadosi S, Liu G (1996) Improved designs for dose-escalation studies using pharmacokinetic measurements. Statistics in Medicine 15(15): 1605-1618.

12. Babb JS, Rogatko A (2001) Patient specific dosing in a phase I cancer trial. Stat Med 20(14): 2079-2090.

13. O'Quigley J, Paoletti X (2003) Continual reassessment method for ordered groups. Biometrics 59(2): 430-440. 
14. Yuan Z, Chappell R (2004) Isotonic designs for phase I cancer clinical trials with multiple risk groups. Clin Trials 1(6): 499-508.

15. Ivanova A, Wang K (2018) Bivaqriate isotonic design for dose-finding with ordered groups. Stat Med 25(12): 2018-2026.

16. Thall P, Nguyen H, Estey E (2008) Patient-specific dose finding based on bivariate out-comes and covariates. Biometrics 64(4): 1126-1136.

17. Guo B, Yuan Y (2017) Bayesian phase I/II biomarker-based dose finding for precision medicine with molecularly targeted agents. Journal of the American Statistical Association 112: 508-520.
18. Indahl U, Liland K, Nas T (2009) Canonical partial least squares unified PLS approach to classification and regression problems. Journal of chemometrics 23: 495-504.

19. Liu S, Guo B, Yuan Y (2017) A Bayesian phase I/II trial design for immunotherapy. Journal of the American Statistical Association.

\section{Your next submission with Juniper Publishers} will reach you the below assets

- Quality Editorial service

- Swift Peer Review

- Reprints availability

- E-prints Service

- Manuscript Podcast for convenient understanding

- Global attainment for your research

- Manuscript accessibility in different formats (Pdf, E-pub, Full Text, Audio)

- Unceasing customer service

Track the below URL for one-step submission https://juniperpublishers.com/online-submission.php 